\title{
Service-users' experiences of an early intervention in psychosis service: An interpretative phenomenological analysis
}

\author{
Katy Harris ${ }^{1} *$, Christine Collinson ${ }^{2}$ and Roshan das Nair ${ }^{3}$ \\ Early Detection and Intervention Team, Early Intervention Service, Birmingham \\ and Solihull Mental Health NHS Foundation Trust, UK \\ 2Early Intervention in Psychosis Service, Nottinghamshire Healthcare NHS Trust, \\ UK \\ Institute of Work, Health and Organisations, University of Nottingham, UK
}

\begin{abstract}
Objectives. Previous research regarding Early Intervention in Psychosis (EIP) services has mainly adopted quantitative methodologies, in order to study the effectiveness of EIP services. Research studies that have explored service-users' experiences of EIP services are small in number. This research aimed to explore service-users' experiences of being in contact with an EIP service, its impact of their experience of psychosis and current life situation.

Design. Interpretative Phenomenological Analysis (IPA) was used to conduct an in-depth qualitative study of a small sample of EIP service-users, in order to explore their experiences of being in contact with the service.

Method. Flexibly guided interviews were conducted with eight service-users who had been receiving a service from an EIP team for more than 2 years and were recruited using a purposive sampling method. Verbatim interview transcripts were analysed using IPA.
\end{abstract}

Results. Five super-ordinate themes, developed from the analysis, are discussed in sections: Stigma, Relationships, Understanding the experiences, Sense of agency, and Impact on sense of self. Sub-themes of these super-ordinate themes are also discussed.

Conclusions. The themes developed from the analysis were envisioned as representing an overarching theme of 'A personal journey of recovery', which was influenced by participants' involvement with the EIP service. Clinical implications include the need for EIP services, as with other mental health services, to find ways to promote recovery and create opportunities for agency and control. Future research directions are also discussed.

\footnotetext{
* Correspondence should be addressed to Dr Katy Harris, Early Detection and Intervention Team, Early Intervention Service, Birmingham and Solihull Mental Health NHS Foundation Trust, I Miller Street, Aston, Birmingham B6 4NF, UK (e-mail: kateharris84@hotmail.co.uk).
} 
Early. Intervention in Psychosis (EIP) services are specialist multidisciplinary teams, working with individuals aged 14 and 35, who experience their First Episode of Psychosis (FEP) and are an integral part of UK mental health services (Department of Health [DoH], 2000, 2001). EIP services are also known nationally by alternative names such as Early Interventions Services (EIS) or Early Intervention Teams (EIT). These services focus on reducing delay in receiving intervention for early psychosis and providing sustained intervention throughout the early phase (Reading \& Birchwood, 2005). EIP services were developed following the 'critical period' hypothesis (Birchwood, Todd, \& Jackson, 1998) and take an optimistic view of recovery, aiming to address the symptoms of psychosis and the social context, whilst also considering how it is experienced by the individual (Sainsbury Centre for Mental Health, 2003).

Early access is crucial to the EIP model, which includes an assertive outreach approach to engagement and removing service barriers (DoH, 2001). Therefore, initial research focused on reducing delay in the initiation of FEP treatments (e.g., Marshall et al., 2005), however, this highlighted difficulties in accurate measurements and definitions of duration of untreated psychosis. Other research focusing on the effectiveness of EIP services (e.g., Bertelsen et al., 2008; Garety, Craig, \& Dunn, 2006), have demonstrated the positive effect of these specialist service interventions on a range of symptoms and outcomes. However, demonstrating these effects over longer time periods is still an ongoing research area.

Although quantitative studies aim to establish whether or not services are effective, qualitative research is important in understanding why an intervention is effective (Medical Research Council [MRC], 2000). Qualitative research aims to understand peoples' experiences as they encounter and live through the events (Elliott, Fischer, \& Rennie, 1999) and attempts to interpret the meanings people bring to them (Denzin \& Lincoln, 2005).

Previous qualitative studies have explored peoples' subjective experiences of psychosis, how individuals recognize and respond to psychosis (Hirschfeld, Smith, Trower, \& Griffin, 2005; Judge, Estroff, Perkins, \& Penn, 2008), personal experiences of hope (Perry, Taylor, \& Shaw, 2007), and recovery (e.g., Ridgway, 2001). In the recent National Institute for Health and Clinical Excellence (NICE) publication (2009), narratives from people with a diagnosis of schizophrenia discussed the importance of good relationships with mental health professionals and service-user agency in treatments decisions.

There is a paucity of qualitative research exploring service-users' experiences of EIP services. O'Toole et al. (2004) conducted a focus group evaluation of service-users' experiences of a UK specialist intervention for FEP. Positive views included the 'human' approach and involvement in decision making. However, the authors recognized the potential for bias due to the inclusion of self-selected participants. It is also suggested focus groups have a tendency to produce consensus and may not be ideal for exploring individual experiences (Newton, Larkin, Melhuish, \& Wykes, 2007). However, more recent publications have begun to discuss methods in which Interpretative Phenomenological Analysis (IPA) can be effectively applied to focus group data, in order to explore personal experiences (Palmer, Larkin, Visser, \& Faden, 2010).

Larsen (2007) used a person-centred ethnographic approach to study a Danish EIP service. The service was seen to offer support and explanations, which helped serviceusers understand their difficulties. Both similarities and differences in the recovery models and therapeutic interventions offered by different staff were identified. However, this study only offers limited insights into a UK model, as the author acknowledged the inevitable contextual differences between Danish and UK services. 
Other studies have either focused on service users with psychosis, from non-specialist services (e.g., community mental health teams prior to the establishment of a specialist EIP service; Barker, Lavender, \& Morant, 2001; McKenzie, 2006) or have reported limited qualitative data from non-UK EIP services (Theuma, Read, Moskowitz, \& Stewart, 2007). EIP services adopt a recovery-focused approach aiming to positively impact on serviceusers lives and views of their experiences, whilst also reducing stigma associated with psychosis and barriers to accessing services (DoH, 2001). Due to the specialist team delivery and recovery focus and the small number of qualitative studies identified, a more in-depth understanding of service-users' experiences of a UK EIP service approach was required. Therefore, the aim of this research was to

- Explore what is it like from a service-users perspective to be in contact with an EIP service (UK model), using an in-depth qualitative research method.

- Specifically, to explore how being in contact with the service has impacted on their view of their psychosis and their current life experiences.

\section{Method}

\section{Participants}

Participants were recruited from an NHS EIP service, comprising of two multidisciplinary teams (including a psychologist, psychiatrists, and community psychiatric nurses) covering both the city and county. The service was established in 2005, in line with national guidelines (DoH, 2001) and provides a 3-year service to individuals aged 18-35.

Eight participants were recruited, using a purposive sampling method, and had all been in the EIP service for between 2 years and 2 years and 11 months (due to changes in service provision in the last month, as the EIP service is 3-year service). Those with the longest time in the service were approached first, as they had the most experience of the EIP service. The IPA (Smith \& Osborn, 2003) process was optimized by only including participants who could attend the interview without the need for an interpreter.

\section{Recruitment}

Potential participants who met the inclusion criteria were identified by the EIP clinical psychologist and were approached by their care coordinator (all service-users are allocated a care coordinator who is their primary contact with the service), who provided them with an information sheet. Interested service-users gave verbal consent to be contacted by the lead researcher. Written consent was obtained by the lead researcher prior to the interview.

If for any reason (e.g., impact of medication/symptoms of psychosis on cognition) the service-user was considered not to have capacity to make an informed decision, or was judged to be a significant risk to themselves or others, they were excluded from the study. Full NHS Research Ethical Approval to conduct this research was obtained.

\section{Data collection}

The lead researcher conducted all interviews, which were audio recorded and ranged from 45 to $110 \mathrm{~min}$. Participants were told the purpose of the interview was to discuss 
their experiences of being in contact with the EIP service, and interviews were flexibly guided by an interview schedule.

\section{Analysis}

As participants were given the opportunity to talk about their experiences, the chosen method of analysis was IPA (Smith \& Osborn, 2003). This approach aims to understand how participants make sense of and give meaning to their experiences and is concerned with the individual's personal perceptions of an event, as opposed to producing an objective record. The meanings people attach to their experiences are explored through the researcher engaging in a process of interpretation (Smith \& Osborn, 2003).

Interviews were transcribed verbatim and analysed by the lead researcher, using the IPA method outlined by Smith and Osborne (2003) as a guide. Each transcript was engaged with separately and initial notes were transformed into emerging themes. Connections between emerging themes were identified to allow the combination of separate themes into superordinate themes. A master list of themes was created for each participant and newly emerging themes were compared against earlier transcripts. Themes from interviews were combined to construct a final table of superordinate themes.

\section{Quality assurance}

As IPA relies on the researcher's interpretation of the data, it is important to ensure the interpretations given are as trustworthy and credible as possible.

Standards for conducting good qualitative research were applied, where appropriate (Elliott et al., 1999; Lincoln \& Guba, 1985). In order to show trustworthiness, the lead researcher aimed to be as transparent as possible throughout the analysis and in reporting the results. Therefore, direct quotations were used to ground themes within the text. A reflective research diary was utilized to create an audit-trail of the analysis process and to produce a critical reflective discussion. The reflective diary was used to identify the lead researchers pre-existing assumptions and their role and influence in the interpretation process (Elliott et al., 1999). These reflective processes were important, as the lead researcher had previously worked in an EIP service and had their own experiences and beliefs about these services.

\section{Results}

Five superordinate themes were developed from the interviews (1) Stigma; (2) Relationships; (3) Understanding the experiences; (4) Sense of agency; (5) Impact on sense of self. All identifying features in quotations have been altered to maintain anonymity (e.g., pseudonyms). The participants demographic and service details (Table 1) were provided by care coordinators.

\section{Stigma}

This superordinate theme captures participants' descriptions of the multifaceted nature of the stigma related to their experiences, including self-stigma, others'judgements, and stigma of services. 
Table I. Participants demographic and service details

\begin{tabular}{lclll}
\hline Participant & Age & Gender & \multicolumn{1}{c}{ Ethnicity } & Length of time in EIP service $^{\text {a }}$ \\
\hline 1 & 37 & Male & White and Black Caribbean & 2 years 11 months \\
2 & 37 & Male & White British & 2 years 10 months \\
3 & 32 & Male & White British & 2 years 7 months \\
4 & 21 & Female & White British & 2 years 9 months \\
5 & 23 & Male & White British & 2 years 8 months \\
6 & 31 & Female & White British & 2 years 9 months \\
7 & 24 & Male & White and Asian & 2 years 9 months \\
8 & 29 & Female & White and Asian & 2 years 10 months \\
\hline
\end{tabular}

aLength of time since referral was accepted by the EIP service.

\section{Self-stigma}

This captures the participants' rich accounts of personal shame and self-judgement about their and other peoples' experiences of psychosis. The following extract introduces this issue, as the participant expresses her distinction between different psychiatric diagnoses, which was echoed by other participants:

P8: ... I had psychosis, so I didn't like that. I mean depression's fine with me, but having psychosis isn't.

... to me it means that it's something whacky and totally... serious and ... well ...

I'm just ashamed of it really...

This extract illustrates what was interpreted as an implicit hierarchy of psychiatric diagnoses, in which psychosis was deemed to be more serious and shameful than other diagnoses.

Participants also described how their own personal shame impacted on their ability to talk to people about their early experiences of psychosis, which was interpreted as an implied barrier to accessing the EIP service:

P5: ... well I couldn't talk to her (Girlfriend) ... .

Interviewer: You said you couldn't talk to her about it?

P5: No because you just sound ... too weird...

\section{Others' judgements}

Participants described their experiences of other people's judgements about their diagnoses and behaviours and how these initially impacted on their willingness to talk about their experiences. This was interpreted as illustrating a link between the participants' personal feelings of shame and the judgments made by others. Collectively, these were seen as creating a barrier to the EIP service.

Participants gave multiple examples of ongoing feelings of being misunderstood and judged by others outside of the relationships formed through the EIP service. One participant explicitly connected her parents' shame to her own personal shame:

P8: ... I guess that wore off on me, made me a bit erm ... ashamed as well. Maybe I wouldn't have been as ashamed of it as ... I am if my parents hadn't been sort of the instigators of the shame... 


\section{Stigma of services}

Accounts suggested a pre-existing stigma surrounding traditional mental health services, with them representing power and control over people. This stigma was seen as being projected onto the EIP service and contributed to initial anxieties about their involvement. More specifically, participants spoke about the impact of the EIP services name on their feelings of shame and separation:

P8: ... the fact that it's not known and it's this little ... little... specialised service ... and your sort of in this group and you're not in sort of the mainstream I guess. It's the way I feel of it, it might very well be mainstream but I, I feel as though it's not, I feel as though it's some kind of little special group that needs to be taken aside cus they need that extra remedial lesson type thing, like in school when you have to be taken out of class to go to remedial lessons.

This participant highlights her distinction between mainstream services and EIP and implies this can create feelings of separation from other people with mental health problems. This was interpreted as an extension to discussions of an implicit hierarchy, by suggesting a hierarchy within mental health services, with some being more accepted than others.

In contrast, some participants discussed the powerful impact of the EIP service approach on reducing the stigma associated with mental health services:

P7: ... that's what EIP kind of does, it softens that relationship between mental health authority and the punters so to speak who use that service, cus they... I speak for myself, they did ... they did change the way I think about it ...

Accounts suggested a need for the public and professionals to be educated and knowledgeable about EIP services, as disseminating knowledge was viewed as a way of battling stigma.

\section{Relationships}

Participants spoke in-depth about relationships that had assumed importance to them during their time in the EIP service.

\section{Peer-support}

Participants' described their experiences of attending staff facilitated EIP groups (within the EIP service and through service links with external agencies), which offer peer support for people with psychosis or other mental health problems. All participants who had attended these groups stated they had been offered the opportunity by their care coordinator.

Participants acknowledged the role of these groups in reducing feelings of social isolation:

P1: ... a little coffee bar where a lot of ex-patients can come back in a see people and you get talking and that's helped me because I live alone and I, my parents live in (another country), so there's times when I don't really get to see anyone ... .

Accounts suggested that in contrast to feeling judged and misunderstood, the relationships developed in the groups provided an opportunity to feel understood and created a sense of belonging. This belonging was interpreted as a vehicle to overcome feelings 
of shame and also instilled confidence in the participants. For example, the following participant's story about a peer-group boat trip, illustrates how others' openness acted as a catalyst to reducing his personal shame:

P5: ... there we're all these other people and said like oh why, what are you doing here? and one person says, I says, "oh we're a youth club" or something and ... he goes "no we're not, were all loons, we've got mental health problems".

Interviewer: How did you feel when he said it?

P5: I felt like quite ... liberated in way, just like, yeah that's me ...

It was also interpreted that the groups provided an opportunity for participants to take on the role of the expert by becoming a 'helper' for others and is seen as over-lapping with the theme Sense of agency:

P1: ... people tell you what their illness is and what kind of, then you try to come up with something that will help them and they do it vice versa to you ...

\section{Care coordinator relationship}

The superordinate theme 'Relationships' also captures the nature of the participants' relationships with their EIP care coordinators. In participants' accounts, there was a consistent theme that care coordinators represented the face of the service. This was interpreted as illustrating the importance of the relationship between participants and care coordinators, on the participants' views of the EIP service.

For many participants, this relationship was their primary channel to support, knowledge about psychosis and recovery, involvement with other aspects of the EIP service, and external services. Accounts suggested a sense of the participants often adopting the views of their care coordinators and a willingness to extend their trust for their care coordinator to others, in this participant's case accepting psychology involvement:

P1:... I've been involved seeing Sam (Care co-ordinator) every fortnight or so, or every month, or however it seems to go for the time. And erm that was going well, so I thought well she mentioned it and I err, I, I, liked Sam, and I thought she's trying to help you so why not, I'll give it a go...

\section{Understanding the experiences}

This superordinate theme encapsulates the participants' attempts to understand their experiences of psychosis and how it was influenced by their involvement with the EIP service.

Participants discussed their explanations of why they had experienced psychosis, with childhood experiences, stress or societal pressures, all being examples of speculation regarding predisposing and precipitating factors. For some, their experience of being in contact with the EIP service was seen as a necessary experience in order to move forward as a person, which is connected to the theme Impact on sense of self:

P2: ... perhaps you thought you had got over things and that but perhaps its time to get things out the files and look through it and rectify everything and put you back to square one again, break you down to build you up again. 
Participants were interpreted as normalizing their experiences, by identifying commonalities between themselves and other people who had experienced psychosis. Specifically, accounts acknowledged how the EIP service influenced this normalization, as participants suggested early contacts with the service provided them with information about psychosis and their first sense of relief and optimism about their future:

P5: ... they just told me that the fact was, there are other people like you and you can get better from it ...

... .yeah and that just, relief really and like before I just thought I never, OK the rest of my life not getting better...

This was seen as demonstrating the value of normalization in fostering a deeper sense of hope, regarding their experiences, and the role of EIP in this process.

Beyond fostering a sense of hope, explanations offered by the EIP service also allowed participants to normalize their experiences by drawing comparisons between themselves and other people within the general population. This was interpreted as a way of participants re-identifying with the general public:

P8: Yeah, you know everyone is susceptible...

... now I view it as part of everyone, everyone has the potential to become ill ...

However, some participants normalized their experiences beyond explanations offered directly by the EIP service, towards what was interpreted as more of a spectrum of psychosis, on which everyone is situated to different degrees:

P3: ... I think everyone is in psychosis; it's just what level you get to you know...

This theme highlights the influential role of the EIP service in the participants' understandings of their experiences. However, it also demonstrates that participants were able to form their own personal frameworks for understanding, beyond those offered by the service.

\section{Sense of agency'}

This superordinate theme captures the participants' contrasting accounts of feeling both a passive recipient and an active agent in their experiences.

\section{Acceptance and control}

Participants' accounts were interpreted as representing a shift from an initial avoidance of acknowledging their experiences, towards an acceptance of their presence. Initial contacts with the EIP service encouraged participants to confront their experiences. Whereas, later on, the service provided an opportunity for some participants to talk about the experiences and develop ways of recognizing symptoms, both of which were interpreted as initial steps towards an acceptance and control of their experiences.

When discussing her current situation, one participant used the word 'recovered', which she defined as:

P8: Sort of living with my symptoms and dealing with it, accepting it. 
... I've got chinks in my amour, because not all your body ever can be protected by armour, there's gonna be weak spots and there's gonna be times when you sort of slip and have a bit of wobble.

This extract illustrates the participant's acceptance of her symptoms; an attitude that she suggested had been influenced by discussions with her care coordinator. However, this was interpreted as representing a sense of being resigned to a life with psychosis, and it being something to be endured. In contrast, other participants described a sense of active control over their experiences, which was supported by the EIP service, for example,

P1: ... even if I do hear voices, I know that it's not actually people talking, I know its actually just going off in my own brain ... I'm able to, to think, I can challenge it myself...

... Jane (EIP Psychologist) helped with that as well ...

... when I do hear the voices, I go straight to my list that I got off Jane and it works ...

This sense of agency was elaborated further, as although participants attributed aspects of their progress to the EIP services actions; they also recognized their own role and the importance of personal responsibility for their future:

P3: ... they've (EIP service) offered me the psychology, they've offered me support in every area ... but what they can't do is provide a solution yer see, they can only help, ... there is no one who can actually provide the solution, other than yourself...

\section{EIP service involvement}

This captures an alternative aspect of agency, in which the participants described their varied feelings of control over interventions, during their contact with the EIP service. Accounts suggested, upon entering the service, many participants were offered limited options regarding treatments, with medication being the predominant or sole choice. Participants described feeling pushed into using medication and the negative impacts:

P6: It feels as though... everything is being taken out of your hands and you just ... feel worthless really.

In contrast to feelings of powerlessness over treatments, other participants' accounts suggested a different view of the EIP service approach, as they described a sense of control over the pace and level of involvement:

P7: ... force isn't a remedy, is something that like early intervention go by, like the, they don't force people ... It's up to you how much involvement you have with them ...

One participant explicitly described a dramatic change in her level of control, from a sense of being a passive recipient of EIP services to an active agent, which she described as 


\section{Impact on Sense of Self}

This superordinate theme encapsulates participants' accounts of the impact of their experience of psychosis and their contact with the EIP service, on their view of themselves and their place within the world.

\section{A sense of discovery}

This theme was interpreted as illustrating the participants' experiences of discovering a new and stronger self, following their experience of psychosis and the service. It initially captures participants' positive appraisals of being in contact with the EIP service:

P7: ... not everyone gets caught in the net and like, for me it was good to get caught in the net, because I faced everything...

This positive appraisal of receiving the service was expanded further, as participants' described ways in which they had experienced positive changes in themselves:

P7: ... I'm stronger now than I ever was ...

P3: Because I was never really in touch with my feelings or emotions, things like that, yer know, never really in touch with them ...

Some participants directly acknowledged how the EIP service had helped them to identify positive changes in themselves:

P4: ... Sarah (Care co-ordinator) says ... if I would have been bullied at (names hospital), when I came out of prison I probably would have smacked them back, I probably would have beat them up for just looking at me ... and so I've calmed right down in that sense...

\section{Place within the world}

Due to their psychosis, many participants had experienced dramatic changes in their lives, resulting in feelings of a detachment from their previous world. However, participants identified the ways in which the EIP service had supported them in trying to re-establish vocational and social aspects of their lives:

P8:... I'm gonna start a new job on Monday err ... yeah I got married whilst being in services, in EIP ...

... I've been through a lot with sort of EIP propping me up really, so that's good.

P1: ... with the help again of Sam (Care co-ordinator) ... I keep in contact with friends and go round and see them and ask them round to come and see you ...

Conversely, some participants described a deeper and ongoing sense of detachment from their world. In particular, one participant discussed his feelings of incompatibility with the world around him and a lack of understanding for his new found sense of self:

P3: ... all the psychotic people are operating on the same frequency and all the people outside of that are operating on another frequency... 
This highlights that although participants identified positive changes and the role of EIP in helping to re-establish aspects of their lives, for some, there was a deeper sense of incompatibility with the world, which was not resolved by their contact with the EIP service.

\section{Discussion}

This study aimed to explore service-users' subjective experiences of being in contact with an EIP service and its impact on their experience of psychosis. Flexibly guided interviews were conducted with eight EIP service-users and five superordinate themes were developed.

The themes identified can be interpreted as representing an overarching theme of 'A personal journey of recovery', which moved beyond symptom alleviation or management and was interpreted as being influenced by involvement of the EIP service. For participants, the journey involved overcoming stigma associated with psychosis and mental health services, normalizing and developing an understanding of and accepting their experiences, gaining a sense of agency and control, discovering and trying to assimilate a new self-concept.

The elements of thisjourney are not interpreted as linear stages; as necessary, or being achieved by everyone. Within the literature, there have been attempts to propose stage models of recovery from psychosis and other mental health problems (e.g., Andresen, Oades, \& Caputi, 2003; Young \& Ensing, 1999). However, stage models could be viewed as incongruent with 'consumer models' of recovery and personal narratives (Bellack, 2006; Ridgway, 2001), in which psychosis and recovery are viewed as non-linear, personalized and part of an ongoing journey.

Aspects of thisjourney have been previously identified within the literature, including the multifaceted nature of stigma (Dinos, Stevens, Serafty, Weich, \& King, 2004; Judge et al., 2008). However, participants in this study discussed the stigmatizing effect of the EIP service specifically. Accounts suggested that initially the EIP service's name had a powerful impact on their feelings of shame and separation from other people with mental health problems. Attitudes represented what was interpreted as an implied hierarchy of mental health services, where mainstream services were initially viewed as more acceptable than EIP. However, once participants had engaged with the service, their accounts went on to highlight the powerful role EIP played in battling against the stigma of mainstream mental health services. This has clinical implications for EIP services, as it suggests staff need to continue to be aware of the stigmatizing nature of a specialist service, whilst continuing to ensure that the identified and well-recognized benefits of the specialist EIP service are maintained and promoted. EIP services need to take a multifaceted approach to overcoming stigma, in order to tackle self-stigmatizing attitudes and increase the public's knowledge of specialized services.

The theme Relationships highlighted the influential role of staff-facilitated EIP groups that offer peer-support. Participants' experiences of this peer-support were consistent with previous research (Newton et al., 2007; Perry et al., 2007) and theories of group therapy, which emphasize the emotional release that can occur when experiences are normalized (Yalom, 1985). However, this current research offers an insight into the experiences of peer groups from service-users in an EIP service, which has not been previously studied. EIP services aim to provide an opportunity for service-users to attend peer groups (DoH, 2001), and this research suggests the potentially important 
and influential role of these groups on people's journey of recovery. However, the appropriate methods of promoting and delivering these groups were not explored and could be studied in future research.

Many other elements of participants' journeys are consistent with research that has explored important aspects of recovery from mental health problems. This includes the importance of personal frameworks for understanding (Ridgway, 2001), hope (Perry et al., 2007), agency and control (Barker et al., 2001; Young \& Ensing, 1999), and growth, which is a relatively under-explored area in psychosis (Andresen et al, 2003).

This study adds to this literature, as it explored the impact of being in an EIP service and the influential role of staff on this personal journey. The study suggests being in contact with the EIP service, participants were provided with an opportunity to form a personal understanding of their experiences, without necessarily adopting a medical model of psychosis. Some participants' descriptions were interpreted as being congruent with views of psychosis as a continuum, in which authors such as Bentall reject the assumption of '... an unambiguous dividing line between the psychologically bealthy and psychologically disturbed... '(Bentall, 2003, p. 494).

Relationships with care coordinators were seen as influential in the participants' views of the EIP service, their engagement with other services, fostering a sense of hope, impacting on their personal understanding, their sense of agency, and sense of self. Previous research has noted a tendency for participants to talk specifically about their care coordinators when referring to EIP services (O'Toole et al., 2004) and has suggested the influence of EIP staff views and professional backgrounds on the explanation of psychosis offered to clients (Larsen, 2007). However, the current research also suggested support offered by the EIP service had potential limits and was unable to resolve all the complex layers of the participants' ongoing journeys.

\section{Clinical implications}

The important and influential nature of the relationship between service-users and EIP staff and the impact of the service on their personal journeys, has important clinical implications. EIP services need to consider the personal and professional attitudes, values and behaviour of staff throughout recruitment, training, and supervision. Care coordinators and other staff need to be supported by the EIP service to develop a conscious awareness of their potentially powerful influence and exercise it with care to promote recovery and provide opportunities for agency.

The importance of developing a sense of agency has further clinical implications, as mental health services operate within the Mental Health Act (2007), a powerful piece of legislation that can remove individual power and agency. Coercion within mental health services can operate at more subtle levels (e.g., Lutzen 1998) and block the development of personal agency. EIP services, as with other mental health services, need to find ways of creating opportunities for personal agency, and where possible, aim to prevent the need for sectioning under the Mental Health Act (2007).

\section{Limitations}

This study's methodology allowed an in-depth engagement with the topic at a level that would have been difficult with less idiographic approaches. However, a potential limitation was that service-users were initially approached by care coordinators and were still engaged with the service at the time of the interviews. Due to the nature of 
these relationships, this may have influenced decisions to participate and unintentionally excluded those with more difficult experiences. However, the participant information sheet explained procedures for confidentiality, in an attempt to minimize this influence. A further potential limitation was the recruitment of participants from one EIP service. It is important to acknowledge that the particular ethos of this service will have also influenced participants' experiences.

\section{Future Research}

Due to the influential role of the EIP service on participants' experiences, future research could explore care coordinators experiences of fulfilling this position in an EIP service, in order to illuminate this relationship further and identify training needs. Secondly, further research could study peoples' experiences after exiting an EIP service, which could allow exploration of peoples' journeys beyond EIP involvement.

\section{References}

Andresen, R., Oades, L., \& Caputi, P. (2003). The experience of recovery from schizophrenia: Towards an empirically validated stage model. Australia and New Zealand Journal of Psychiatry, 37, 586-594.

Barker, S., Lavender, T., \& Morant, N. (2001). Client and family narratives on schizophrenia. Journal of Mental Health, 10(2), 199-212.

Bellack, A. S. (2006). Scientific and consumer models of recovery in schizophrenia: Concordance, contrasts and implications. Schizophrenia Bulletin, 32(3), 432-442.

Bentall, P. (2003). Madness explained. Psychosis and human nature. London, UK: Penguin Books.

Bertelsen, M., Jeppesen, P., Petersen, L., Thorup, A., Ohlenschloeger, J., Le Quach, P.,...Nordentoft, M. (2008). Five-year follow-up of a randomised multicentre trial of intensive early intervention vs. standard treatment for patients with a first episode of psychotic illness: The Opus trial. Archives of General Psychiatry, 65(7), 762-771.

Birchwood, M., Todd, P., \& Jackson. (1998). Early intervention in psychosis: The critical period hypothesis. British Journal of Psychiatry, 172(33), 53-59.

Denzin, N. K., \& Lincoln, Y. S. (2005). The sage handbook of qualitative research (3rd ed.). London, UK: Sage Publications.

Department of Health. (2000). The NHS plan: A plan for investment: A plan for reform. Department of Health, London. Retrieved from www.dh.gov.uk

Department of Health. (2001). The mental health policy implementation guide. Department of Health, London. Retrieved from www.dh.gov.uk

Dinos, S., Stevens, S., Serafty, M., Weich, S., \& King, M. (2004). Stigma: The feelings and experiences of 46 people with mental illness: Qualitative study. British Journal of Psychiatry, 184, 176- 181.

Elliott, R., Fischer, C. T., \& Rennie, D. L. (1999). Evolving guidelines for publication of qualitative research studies in psychology and related fields. British Journal of Clinical Psychology, 38, 215-229.

Garety, P., Craig, T. K. J., \& Dunn, G. (2006). Specialised care for early psychosis: Symptoms, social functioning and patient satisfaction. Randomised controlled trial. British Journal of Psychiatry, 188, 37-45.

Hirschfeld, R., Smith, J., Trower, P., \& Griffin, C. (2005). What do psychotic experiences mean for young men? A qualitative investigation. Psychology and Psychotherapy: Theory, Research and Practice, 78, 249-270. 
Judge, A. M., Estroff, S. E., Perkins, D. O., \& Penn, D. L. (2008). Recognising and responding to early psychosis: A qualitative analysis of individual narratives. Psychiatric Services, 59(1), 96 99.

Larsen, J. A. (2007). Understanding a complex intervention: Person-centred ethnography in early psychosis. Journal of Mental Health, 16(6), 333-345.

Lincoln, Y. S., \& Guba, E. G. (1985). Naturalistic inquiry. London, UK: Sage Publications Ltd.

Lutzen, K. (1998). Subtle coercion in psychiatric practice. Journal of Psychiatric and Mental Health

Nursing, 5, 101-107.

Marshall, M., Lewis, S., Lockwood, A., Drake, R., Jones, P., \& Croudace, T. (2005). Association between duration of untreated psychosis and outcome in cohorts of first episode patients. Archives of General Psychiatry, 62, 975-983.

McKenzie, L. H. (2006). Service-users and carers' experiences of a psychosis service. Journal of Psychiatric and Mental Health Nursing, 13, 636-640.

Medical Research Council. (2000). A framework for development and evaluation of RCT's for complex interventions to improve bealth. London: MRC. Retrieved from www.mrc.ac.uk Mental

Health Act of 2007, c.12 (2007). Retrieved from http://www.opsi.gov.uk/acts/acts2007/ ukpga 20070012 en 1

National Institute for Health and Clinical Excellence. (2009). Schizophrenia: Core interventions in the treatment and management of schizophrenia in primary and secondary care (Update). NICE: London. Retrieved from www.nice.org.uk

Newton, E., Larkin, M., Melhuish, R., \& Wykes, T. (2007). More than just a place to talk: Young people's experiences of a group psychological therapy as an early intervention for auditory hallucinations. Psychology and psychotherapy: Theory, research and practice, 80, 127-149.

O’Toole, M. S., Ohlsen, R. I., Taylor, T. M., Purvis, R., Walters, J., \& Pilowsky, L. S. (2004). Treating first episode psychosis- The service-users' perspective: A focus group evaluation. Journal of Psychiatric and Mental Health Nursing, 11, 319-326.

Palmer, M., Larkin, M., de Visser, R., \& Fadden, G. (2010). Developing an interpretative phenomenological approach to focus group data. Qualitative Research in Psychology, 7(2), 99121.

Perry, B. M., Taylor, D., \& Shaw, S. K. (2007). "You've got to have a positive state of mind": An interpretative phenomenological analysis of hope and first episode psychosis. Journal of Mental Health, 16(6), 781-793.

Reading, B., \& Birchwood, M. (2005). Early intervention in psychosis: Rationale and evidence for effectiveness. Disease Management and Health Outcomes, 13(1), 53-63.

Ridgway, P. (2001). Restorying psychiatric disability: Learning from first person recovery narratives. Psychiatric Rebabilitation Journal, 24(4), 335-343.

Sainsbury Centre for Mental Health. (2003). A window of opportunity: A practical guide for developing early intervention in psychosis services. Briefing 23. Retrieved from www.scmh. org.uk

Smith, J. A., \& Osborne, M. (2003). Interpretative phenomenological analysis. In J. A. Smith (Ed.), Qualitative psychology: A practical guide to methods (pp. 51-80). London, UK: Sage Publications Ltd.

Theuma, M., Read, J., Moskowitz, A., \& Stewart, A. (2007). Evaluation of a New Zealand early intervention service for psychosis. New Zealand Journal of Psychology, 36, 136-145. Yalom, I.

(1985). The theory and practice of group psychotherapy. New York, NY: Basic Books. Young, S. L., \&

Ensing, D. S. (1999). Exploring recovery from the perspectives of people with psychiatric disabilities. Psychiatric Rehabilitation Journal, 22(3), 219-231. 